\title{
Multi-stage four-quadrant phase mask: achromatic coronagraph for space-based and ground-based telescopes
}

\author{
R. Galicher ${ }^{1,2}$, P. Baudoz ${ }^{2}$, and J. Baudrand ${ }^{2}$ \\ 1 Luth, CNRS, Observatoire de Paris, 5 place Jules Janssen, 92195 Meudon, France \\ e-mail: raphael.galicher@obspm. fr \\ 2 Lesia, Observatoire de Paris, CNRS and University Denis Diderot Paris 7, 5 place Jules Janssen, 92195 Meudon, France
}

Received 8 February 2011 / Accepted 28 March 2011

ABSTRACT

\begin{abstract}
Context. Less than $3 \%$ of the known exoplanets were directly imaged for two main reasons. They are angularly very close to their parent star, which is several magnitudes brighter. Direct imaging of exoplanets thus requires a dedicated instrumentation with large telescopes and accurate wavefront control devices for high-angular resolution and coronagraphs for attenuating the stellar light. Coronagraphs are usually chromatic and they cannot perform high-contrast imaging over a wide spectral bandwidth. That chromaticity will be critical for future instruments.

Aims. Enlarging the coronagraph spectral range is a challenge for future exoplanet imaging instruments on both space- and groundbased telescopes.

Methods. We propose the multi-stage four-quadrant phase mask that associates several monochromatic four-quadrant phase mask coronagraphs in series. Monochromatic device performance has already been demonstrated and the manufacturing procedures are well-under control since their development for previous instruments on VLT and JWST. The multi-stage implementation simplicity is thus appealing.

Results. We present the instrument principle and we describe the laboratory performance for large spectral bandwidths and for both pupil shapes for space- (off-axis telescope) and ground-based (E-ELT) telescopes.

Conclusions. The multi-stage four-quadrant phase mask reduces the stellar flux over a wide spectral range and it is a very good candidate to be associated with a spectrometer for future exoplanet imaging instruments in ground- and space-based observatories.
\end{abstract}

Key words. instrumentation: high angular resolution - techniques: high angular resolution - methods: laboratory methods: numerical

\section{Introduction}

Orbital parameters as well as atmosphere physical characteristics of exoplanets orbiting in the outer part of their stellar systems will put strong constraints on planetary formation models. The sole method for studying these objects is direct imaging, which measures both astrometric and spectro-polarimetric parameters. This method is also a direct way to study planet-disk or planet-planet interactions in multiple systems. Up to now only a dozen exoplanet candidates have been imaged in favorable conditions: bright planets orbiting far from their star (several AU). When trying to detect fainter planets with that technique, high contrast and small angular separation between the star and its planet become critical points. Large telescopes are thus required to reach the angular resolution, and coronagraphs or interferometers are needed to reduce the stellar flux. Adaptive optics and speckle calibrations are also necessary to deal with the speckle noise induced by the Earth's atmosphere and optical aberrations. In a few months, the SPHERE (VLT, Beuzit et al. 2008), GPI (Gemini, Macintosh et al. 2008) and HiCIAO (Subaru, Tamura et al. 2006) instruments will combine coronagraphs and extreme adaptive optics to study planets $\sim 10^{6}$ fainter than their star and measure their spectro-polarimetric characteristics. The main limitation will be the uncorrected atmospheric turbulence by adaptive optics and slowly drifting optical aberrations. For the following generation of high-contrast imaging instruments like EPICS for E-ELT (Kasper et al. 2008) and the TMT planet finder (Macintosh et al. 2006), more accurate adaptive optics and speckle calibrations are planned and coronagraphs with higher efficiency are needed. The objective is to associate coronagraphs and speckle calibrators to reach high contrasts $\left(10^{6}-10^{7}\right.$ stellar attenuations and speckle calibration up to a factor of $10^{2}-0-10^{3}$, Vérinaud et al. 2008) over a large spectral band ( 20\%). Several methods are suggested to build achromatic coronagraphs, especially those that use phase-shift masks (Soummer et al. 2003; Mawet et al. 2005, 2006; Carlotti et al. 2009; Mawet et al. 2011). To achromatize the four-quadrant phase mask (FQPM, Rouan et al. 2000), our team proposed to use several monochromatic devices in series and create the multi-stage four-quadrant phase mask (MFQPM, Baudoz et al. 2008). Because the monochromatic FQPM technology is well under control since the developments made for VLT (Boccaletti et al. 2004) and JWST (Boccaletti et al. 2005), an MFQPM is easy to build. We dedicate the present paper to this achromatic coronagraph. We recall the technique principle in Sect. 2 and present a laboratory performance for visible wide spectral bandwidths (20\% and 30\%) in Sect. 3 as well as pupil shapes for space- (off-axis telescope) and ground-based (E-ELT) telescopes.

\section{Principle}

After describing the FQPM chromaticity limitation in Sect. 2.1, we present the multi-stage FQPM coronagraph principle in Sect. 2.2. 


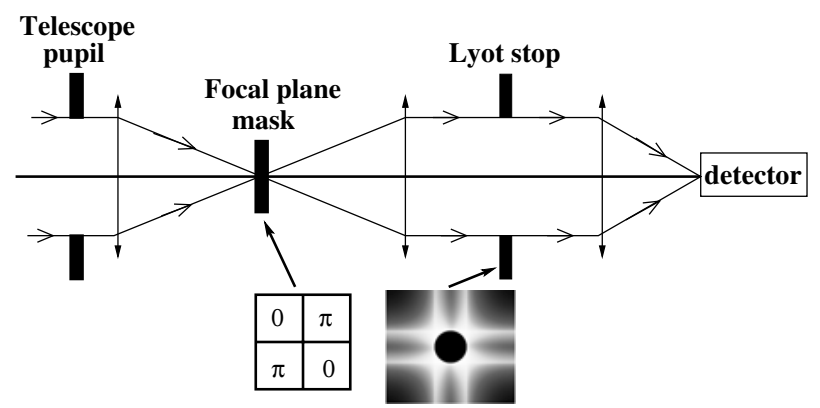

Fig. 1. Single FQPM coronagraph schematics. The image shows the intensity distribution in the pupil plane just before the Lyot-stop.

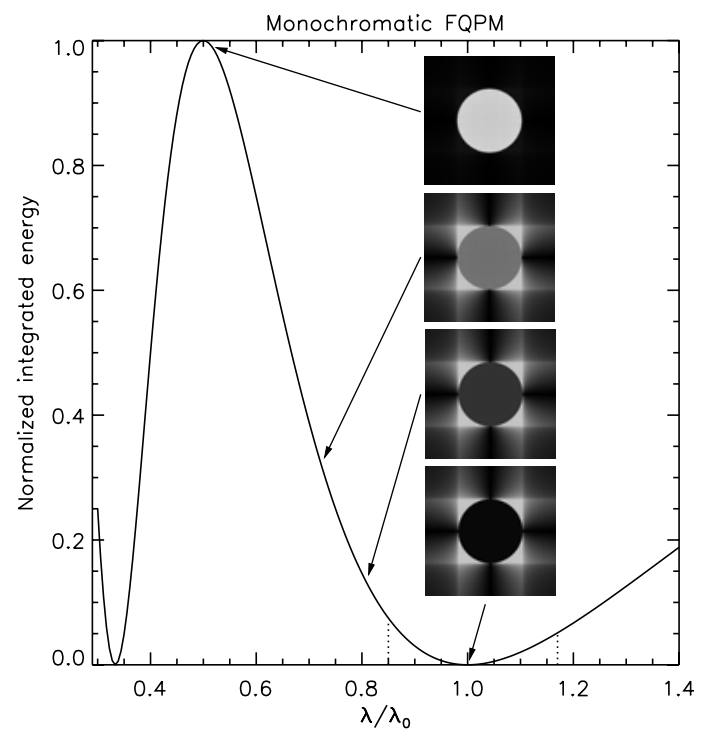

Fig. 2. Integrated energy over the geometrical pupil after a monochromatic FQPM against wavelength. Intensity distributions just before the Lyot-stop are shown for four wavelengths (same gray scale). The curve is normalized to the non-coronagraphic case.

\subsection{Monochromatic FQPM}

We do not present a complete FQPM study in this section. The device is already well described in Rouan et al. (2000), Riaud et al. (2001, 2003), and Boccaletti et al. (2004). We only remark on properties that are used to design the MFQPM.

The FQPM is a phase mask that induces a $\pi$ phase shift on two quadrants of a diagonal and no phase shift in the two other quadrants. In a single FQPM coronagraph (Fig. 1), the on-axis source beam is centered on the phase mask in a focal plane. The resulting diffraction pattern in the following pupil plane is drawn in Fig. 1. No light goes through the geometrical pupil, so that the on-axis source light, called starlight below, is entirely stopped by the Lyot-stop filtering diaphragm. The coronagraph performance mainly depends on the FQPM quality: the $\pi$ phase shift accuracy and the transition thickness between the four quadrants. Phase shifts are usually produced by material steps. This solution makes the mask easy to build but has the disadvantage that the mask is monochromatic because the phase shift is exactly $\pi$ for a unique wavelength, which is called the optimized wavelength. Assuming no wavefront errors, infinitely thin transitions between the quadrants, a full entrance pupil, and no Lyot-stop undersizing, we plot in Fig. 2 the integrated energy over the geometrical pupil plane as a function of wavelength. We normalize the curve to the integrated energy over the

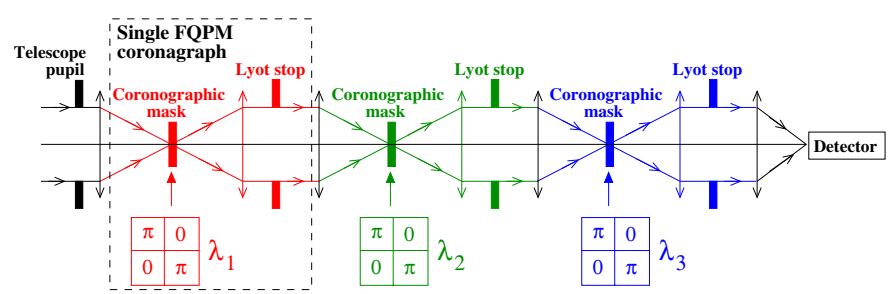

Fig. 3. MFQPM coronagraph schematics. Three monochromatic FQPM are used.

entrance pupil (non-coronagraphic case). The corresponding intensity distributions in the pupil plane before the Lyot-stop are also shown for four wavelengths (same gray scales). At the optimized wavelength $\lambda_{0}$, the entire stellar light is diffracted outside the geometrical pupil and stopped by the Lyot-stop: no startlight goes through the instrument. For other wavelengths $\lambda$, the phase shift is $\pi \lambda_{0} / \lambda$ so that a part of the starlight is not diffracted outside the geometrical pupil and the stellar extinction is not perfect (see pupil images). But this unstopped stellar light is uniformly distributed inside the geometrical pupil in the Lyot-stop plane (Appendix A), which is an interesting feature, as explained in Sect. 2.2. The worst case happens for $\lambda=\lambda_{0} / 2$, because the phase shift is then $2 \pi$ (i.e. 0 ) and the entire starlight energy is inside the geometrical pupil after the FQPM (no attenuation).

We define the average transmission $\tau$ as the ratio of the stellar energy that goes through the Lyot-stop in the spectral bandwidth of interest for the coronagraphic case to the same quantity for the non-coronagraphic case. It represents the average residual stellar energy after the coronagraph. The smaller this number, the more effective the coronagraph. Using a single monochromatic FQPM, $\tau$ is the integrated area under the curve in Fig. 2 bounded by the extremal bandwidth wavelengths and divided by the bandwidth. If the bandwidth is $32 \%$ (vertical dotted lines), $\tau$ is about $2 \times 10^{-2}$. If it is $20 \%, \tau$ is $8 \times 10^{-3}$. These stellar attenuations are not sufficient when exoplanet detections require at least $\tau<10^{-4}$. Several solutions have been proposed to build achromatic FQPM (e.g., Bloemhof 2005; Mawet et al. 2006; Carlotti et al. 2009). The multi-stage four-quadrant phase mask (Baudoz et al. 2008) that we study in this paper is a part of these solutions.

\subsection{Multi-stage FQPM}

Let us consider a single FQPM that perfectly cancels the light at its optimized wavelength $\lambda_{1}$. At another wavelength (i.e. $\lambda \neq \lambda_{1}$ ), the residual starlight is uniformly distributed inside the Lyotstop geometrical pupil. Thus, a second monochromatic FQPM can be used in series to reduce the residual starlight around a second optimized wavelength $\lambda_{2} \neq \lambda_{1}$. After the second stage, the unaffected light by both the first and the second FQPM is still uniformly distributed inside the geometrical pupil. A third stage optimized at $\lambda_{3}$ can be used, and so on. We call this monochromatic coronagraph combination multi-stage fourquadrant phase mask coronagraph (Fig. 3). Note that the combination works because the FQPM unaffected light pupil after the mask is equal to the pupil before the mask up to a factor of proportionality. The following focal mask can then deal with it (Appendix A). Calling $\lambda_{0}$ the central wavelength of the spectral band, we plot the integrated energy in the third Lyot-stop pupil for a MFQPM composed by three single FQPM respectively optimized for $\lambda_{1}=0.987 \lambda_{0}, \lambda_{2}=0.920 \lambda_{0}$ and $\lambda_{3}=1.033 \lambda_{1}$ (blue 


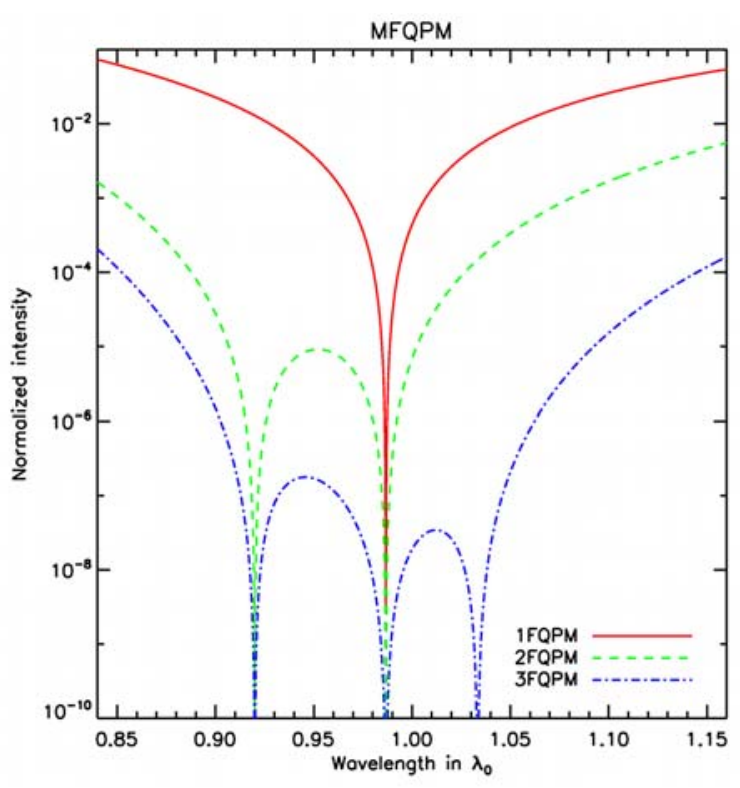

Fig. 4. Residual energy spectra after one (red full line), two (green dashed line), and three (blue dashed-dotted line) monochromatic FQPM. The curves are normalized to the non-coronagraphic case.

dashed-dotted line in Fig. 4). We choose these optimized wavelengths to match the experiment case presented in Sect. 3. We overplot the curves for the first coronagraph used alone (red full line) and associated with the second mask (two FQPM in series, green dashed line). All curves are normalized to the noncoronagraphic case. In both Figs. 2 and 4 we assume no wavefront errors, infinitly thin FQPM transitions, a full pupil and no Lyot-stop undersizing. The vertical scale is now logarithmic, whereas it is linear in Fig. 2. Each monochromatic mask is more effective at its optimized wavelength than at the others. Each mask enhances the contrast over the whole spectral range, and three monochromatic FQPM in series (blue curve) with a full pupil and no aberrations give an average transmission $\tau$ as low as $\sim 10^{-6}\left(\sim 2 \times 10^{-5}\right)$ in a $20 \%(32 \%)$ bandwidth.

\section{Laboratory performance}

This section describes laboratory experiments. We first present the actual MFQPM prototype (Sect. 3.1). We then give the performance using a full pupil (Sect. 3.2) and an obstructed pupil (Sect. 3.3).

\subsection{MFQPM prototype}

A first prototype was used to demonstrate the MFQPM principle using a full circular pupil ( $D$-diameter) a few years ago (Baudoz et al. 2008). The main result was the detection of a $7 \times 10^{-9}$ laboratory companion (i.e., not numerically added a posteriori) at $4.5 \lambda_{0} / D$ in a $20 \%$ visible band after a post-processing differential imaging to enhance the raw MFQPM contrast $\left(7 \times 10^{-7}\right.$ for a signal-to-noise ratio of 3 ). But that prototype worked with a slow beam $(f / 300)$ and was sizable ( $2.5 \mathrm{~m}$ in length). We thus designed a compact prototype with a 40 optical f-number, which is more compatible with a real instrument (Fig. 5). Each FQPM stage is composed of one monochromatic focal mask, one collimating lens, one Lyot-stop and one imaging lens. A translation mechanism moves each stage along the optical axis with a micrometric positioning. The full instrument length is less

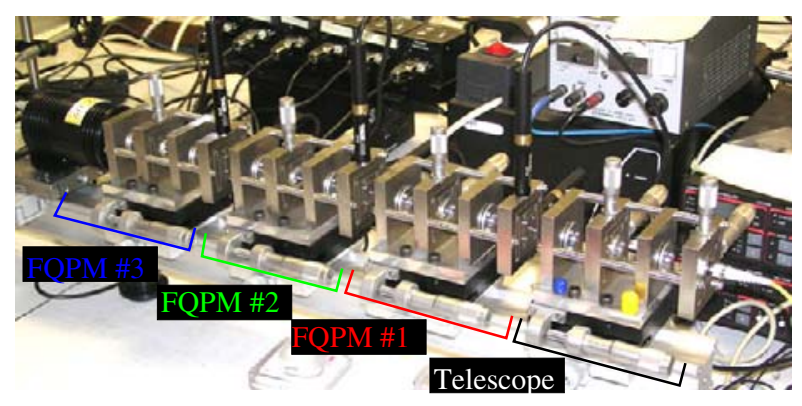

Fig. 5. MFQPM prototype picture. A first stage simulates the telescope and three monochromatic FQPM are in series.

Table 1. Diaphragm diameters for the full pupil experiment.

\begin{tabular}{llll}
\hline \hline Entrance Pupil & $780 \mu \mathrm{m}$ & Lyot 2 & $630 \mu \mathrm{m}$ \\
\hline Lyot 1 & $700 \mu \mathrm{m}$ & Lyot 3 & $570 \mu \mathrm{m}$ \\
\hline
\end{tabular}

Table 2. Experimental performance of actual MFQPM in full pupil.

\begin{tabular}{ccc}
\hline \hline \multirow{2}{*}{$\Delta \lambda$} & \multicolumn{2}{c}{ Average Transmission $\tau$} \\
\cline { 2 - 3 } & On-axis & At $5 \lambda_{0} / D$ \\
\hline $20 \%$ & $1.4 \times 10^{-5}$ & $2.0 \times 10^{-6}$ \\
$32 \%$ & $3.6 \times 10^{-5}$ & $6.1 \times 10^{-6}$ \\
\hline
\end{tabular}

than $45 \mathrm{~cm}(<15 \mathrm{~cm}$ per stage). All lenses are achromatic doublets. Coatings reduce the reflected light to less than $0.1 \%$ in energy from $625 \mathrm{~nm}$ to $825 \mathrm{~nm}$ at each lens surface. The entering beam optical aperture is $f / 40$ at each stage and full or obstructed pupil shapes can be used. The three monochromatic FQPM are optimized at $\lambda_{1}=740 \mathrm{~nm}, \lambda_{2}=690 \mathrm{~nm}$, and $\lambda_{3}=775 \mathrm{~nm}$ (numbering is according to their position from the light source to the detector). Each FQPM transversal position is controlled by two motorized positioning stages. The source creates an ultrabroadband supercontinuum radiation in the visible domain. A beamsplitter is set between the third stage and the detector. One beam converges on the detector (CCD) for imaging. The second beam feeds an optical fiber linked to a spectrometer. The fiber diameter is $\lambda_{0} / D\left(\lambda_{0}=750 \mathrm{~nm}\right)$ and its mount enables accurate movements so that spectra at different positions in the focal plane can be recorded. We use that laboratory setup for both experiments in the full pupil (Sect. 3.2), and the obstructed pupil (Sect. 3.3).

\subsection{Full circular pupil}

We first test the MFQPM prototype with a full circular entrance pupil. A slight Lyot-stop diameter undersizing is required to efficiently block the central source light. We use a $90 \%$ filtering at each stage. The instrument throughput is then $73 \%$. Table 1 gives the diaphragm sizes.

We focus the first study on the spectral MFQPM performance. We record the energy entering the spectrometer fiber when the MFQPM is aligned. We plot that quantity as a function of wavelength when the fiber is centered on the optical axis (black full line in Fig. 6). We normalize the curve to the energy entering the fiber in the non-coronagraphic case (focal masks are pushed off-center). The coronagraph gives an average transmission between $7 \times 10^{-6}$ and $4 \times 10^{-5}$ at each wavelength over a $20 \%$ bandwidth $(660-800 \mathrm{~nm})$. That demonstrates the MFQPM capacity to achromatically reduce the starlight over a wide spectral range. However, the performance (see Table 2) is higher than the expected ones from numerical predictions 


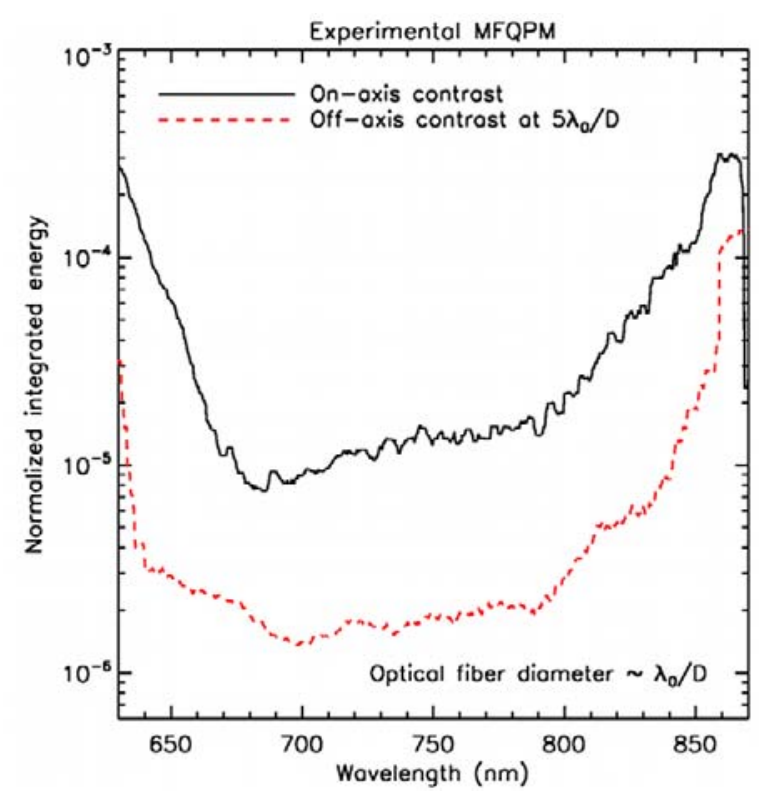

Fig. 6. Experimental residual energy spectra integrated by the spectrometer fiber on the optical axis (black full line) and at $5 \lambda_{0} / D$ (red dashed line) in the focal plane of a MFQPM with a full pupil. The curves are normalized to the non-coronagraphic case.

(Fig. 4, factor up to 10). We investigated the possible actual prototype limitations. We first measured from microscopic images that the transition thickness between the focal mask quadrants is about $1 \mu \mathrm{m}$. We found from numerical simulations developed for previous studies (JWST, Baudoz et al. 2006b) that this thickness set a contrast limit between $10^{-6}$ and $10^{-5}$. Because our bench is not located in a cleanroom environment, the dust has to be taken into account as well to estimate the individual FQPM performance, especially given the small pupil size. A dust density estimation over the pupil area was made using microscopic images at the different surfaces of one doublet lens. We constructed one transmission map per FQPM stage by multiplying all images. These amplitude maps were added in the pupil planes assuming all recorded dusts are utterly dark. We then used our numerical simulations of the complete MFQPM to predict a residual diffracted energy level up to $10^{-5}$ into the fiber. Finally, the midfrequencies errors introduced by the lenses were estimated by measuring surface errors on one lens with a white-light interferometric intrument. Considering the substrate optical index, they reach about $1.45 \mathrm{~nm}$ rms for a single achromatic doublet lens. Even accounting for all lenses, these small phase errors limit the contrast to $10^{-7}$. It seems then that dusts and transitions are the two main current prototype limitations. A careful lens fabrication process and a protective cover over the instrument should be sufficient to decrease the dust effect. We will also work to obtain thinner FQPM transitions.

In the second full pupil MFQPM experiment, the spectrometer fiber is set at $5 \lambda_{0} / D$ from the optical axis and at $45^{\circ}$ from the FQPM transitions (the three mask transitions are parallel). The residual energy spectrum is plotted as a red dashed line in Fig. 6 with the same normalization as the other curve. As expected, the level is lower than for the on-axis case. This level is limited however by speckles that can be detected in the images and that probably come from the limitations described above. A $2 \times 10^{-6}$ planet spectrum could be detected in the raw coronagraphic image with a signal-to-noise ratio (it SNR) of 3 over a $20 \%$ bandwidth. That raw performance will be ameliorated

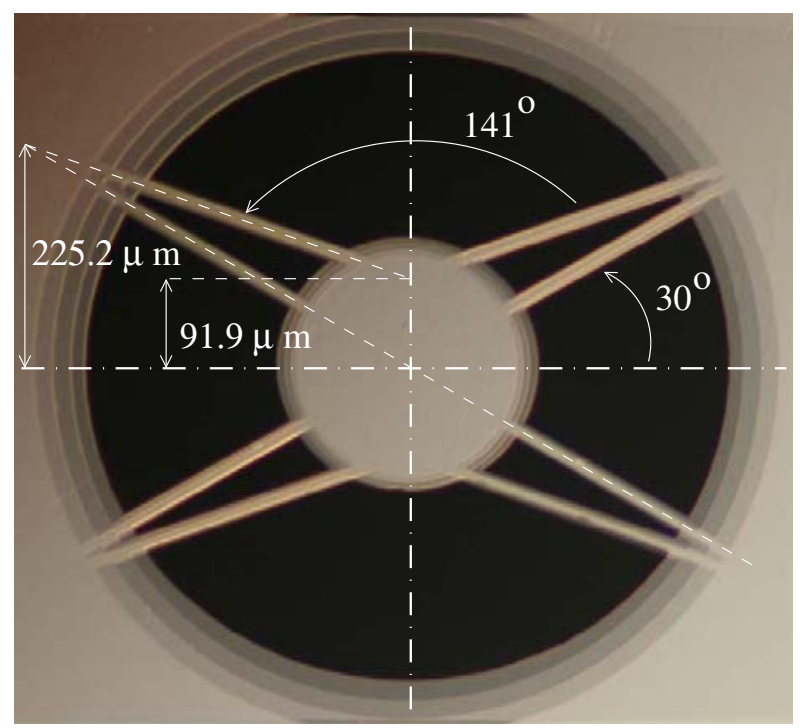

Fig. 7. Superimposition of microscope images of the E-ELT pupil and the three associated $95 \%$ Lyot-stops used in experiment.

Table 3. E-ELT diaphragm parameters.

\begin{tabular}{lccc}
\hline \hline Diaphragm & $\begin{array}{c}\text { Internal } \\
\text { Diameter }(\mu \mathrm{m})\end{array}$ & $\begin{array}{c}\text { External } \\
\text { Diameter }(\mu \mathrm{m})\end{array}$ & $\begin{array}{c}\text { Spider } \\
\text { Thickness }(\mu \mathrm{m})\end{array}$ \\
\hline Telescope & 231 & 780 & 9.3 \\
Lyot 1 & 243 & 741 & 11 \\
Lyot 2 & 257 & 702 & 13 \\
Lyot 3 & 270 & 669 & 15 \\
\hline
\end{tabular}

using the new prototype with less dust and transition effects (under fabrication). A speckle calibration will also enable a contrast gain of up to several magnitudes.

\subsection{Obstructed pupil}

In this section, we report the MFQPM experimental performance when used behind an obstructed telescope. Because the study is realized in the framework of the future European ELT (EELT) planet finder, EPICS (Kasper et al. 2008), the experimental telescope pupil is one of those that are planned for the E-ELT, though our setup does not simulate primary mirror segmentation. We found from numerical simulations made for E-ELT studies that gaps between segments diffract light farther than $30 \lambda / D$ and that their impact is negligible for the coronagraphic performance. The experimental pupil microscope image is presented in Fig. 7. We superimpose in the same figure the three 95\% Lyotstop pictures. All diaphragms are composed of a central obstruction and eight spiders. Both the vertical and horizontal axis are symmetric axes. The spider positions are indicated in the figure, and Table 3 describes diameters and spider thickness. The instrument throughput is $86 \%$ (Lyot-stop undersizing).

Experimental broadband (630 to $870 \mathrm{~nm}$ ) images recorded after aligning no coronagraph, the first FQPM, the two first FQPM, and the three FQPM (complete MFQPM) are presented in the first row in Fig. 8. They are similar to the numerically simulated images drawn in the second row. With no coronagraph, the image is the E-ELT point spread function (PSF) exhibiting a central core and spider diffraction spikes. After a single FQPM, the pattern has a Maltese cross shape. The light whose wavelength is around the phase mask optimized wavelength $\lambda_{1}$ is affected by the coronagraph and is spread in the 


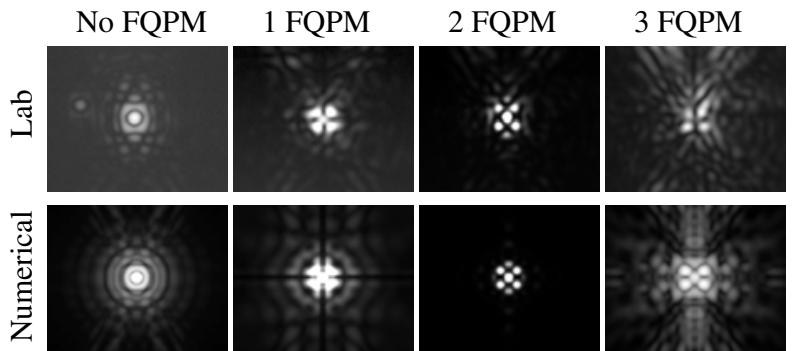

Fig. 8. First row: laboratory images with $0,1,2$, and 3 FQPM in series for an E-ELT pupil. Second row: numerical simulations.
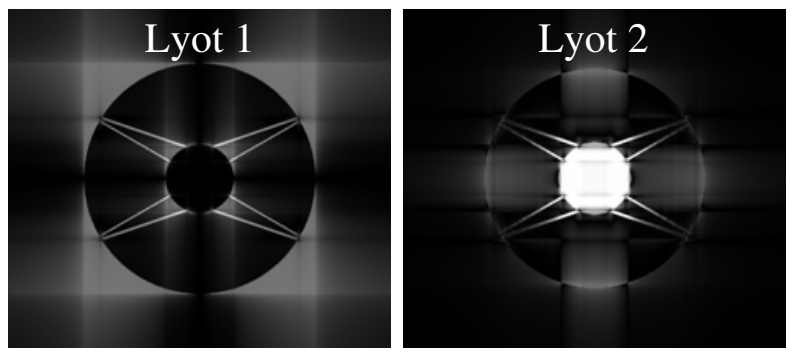

Fig. 9. Energy distribution before the first (left) and the second (right) Lyot-stops in corresponding pupil planes for an obstructed pupil.

cross-braces. The four peak pattern is the FQPM characteristic image when the pupil has a central obstruction. For wavelengths different from $\lambda_{1}$, the light is almost unaffected by the FQPM and it is diffracted in a pattern very similar to the E-ELT PSF: bright central part of the Maltese cross. When a second FQPM is added, it is not easy to distinguish which part of the image caused by light affected or unaffected by the phase masks. It is interesting however to study the energy distribution before the first and the second Lyot-stops in the corresponding pupil planes (Fig. 9). Let us call $D_{0}$ the entrance obstruction diameter. In the first Lyot-stop plane, the pupil obstruction diffraction pattern created by the first FQPM is similar to the $D_{0}$-diameter circular pupil diffraction pattern (Babinet's principle). The image on the left in Fig. 9 shows the light that goes through the first Lyot-stop around the central obstruction. This explains why the single FQPM performance is degraded compared to the case with no obstruction. In the focal plane, the leak creates the four peaks of the Maltese cross (second column in Fig. 8). Consider now that a second FQPM is used in series. It diffracts the light that is diffracted by the first mask, so that in the second Lyotstop plane, the obstruction-diffraction pattern is the obstruction itself (right in Fig. 9). All light that leaks through the first Lyotstop is then blocked by the second Lyot diaphragm obstruction. The same property is used in the multi-stage vortex coronagraph (Mawet et al. 2011). More precisely, a part of the obstruction diffracted light still goes through the second Lyot-stop because the first Lyot-stop filters the obstruction diffraction pattern (finite external diameter), and the first and second focal masks are not optimized for the same wavelengths. The central obstruction bad effect is very well attenuated however, and the MFQPM performance is very attractive even with a $30 \%$ obstruction in diameter as for the E-ELT pupil we used (see below). Finally, like the image produced by a single FQPM, the image recorded after the third FQPM (complete MFQPM, Fig. 8) reveals a four-peak pattern close to the center because a small part of the light is affected by the obstruction of the second Lyotstop (odd number of FQPM). However because the flux was attenuated at all the wavelengths by the instrument, no bright peak remains in the center unlike in the single FQPM case.

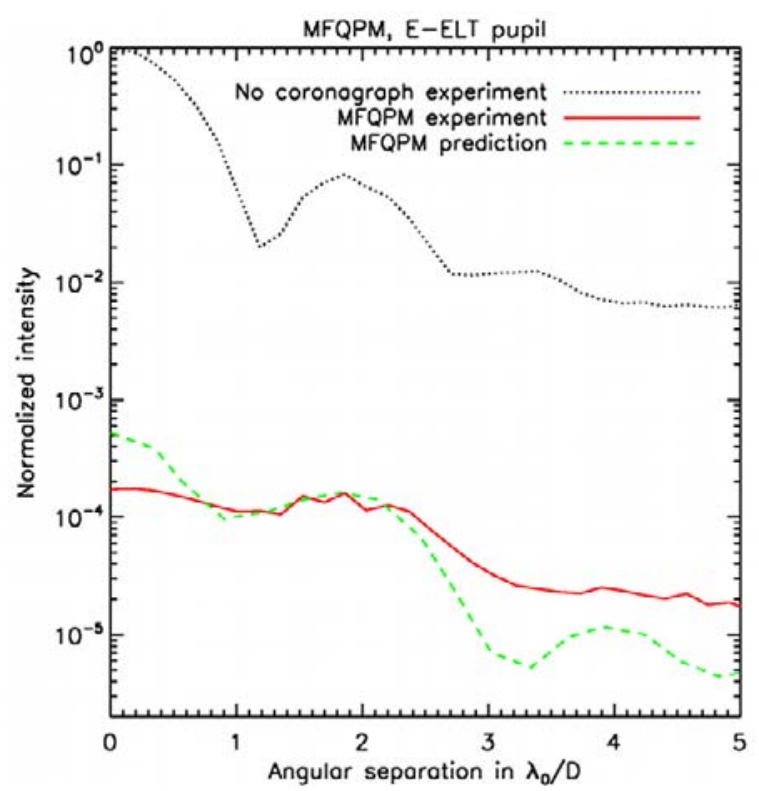

Fig. 10. Experimental intensity against angular separation after no coronagraph (black dotted line) or MFQPM (red full line) in visible (630 to $870 \mathrm{~nm}$.). The numerical prediction is shown in a green dashed line. The curves are normalized to the non-coronagraphic PSF maximum. Entrance pupil is the E-ELT pupil.

Table 4. Current MFQPM experimental performance with E-ELT pupil.

\begin{tabular}{ccc}
\hline \hline \multirow{2}{*}{$\Delta \lambda$} & \multicolumn{2}{c}{ Average transmission $\tau$} \\
\cline { 2 - 3 } & On-axis & At $5 \lambda_{0} / D$ \\
\hline $20 \%$ & $3.2 \times 10^{-4}$ & $6.4 \times 10^{-5}$ \\
$32 \%$ & $3.8 \times 10^{-4}$ & $7.1 \times 10^{-5}$ \\
\hline
\end{tabular}

We also notice that the image quality beyond than $2 \lambda_{0} / D$ is limited by speckles at a level specified in Fig. 10 (experimental curve above predictions made with no speckle noise). The curves represent the experimental image intensity profiles recorded without coronagraph (black dotted line) and after the MFQPM prototype (red full line) against the angular separation to the central source. A numerical simulation prediction is overplotted in a green dashed line (no phase errors and infinitely thin transitions). All curves are normalized to the E-ELT PSF maximum intensity (no-coronagraphic case) and correspond to the intensity integrated over a $32 \%$ band $(630$ to $870 \mathrm{~nm})$. The average transmission $\tau$ in the $32 \%$ band is $\sim 4 \times 10^{-4}$, and from the contrast curve, we find that a $5.9 \times 10^{-5}\left(5.9 \times 10^{-4}\right)$ companion can be detected as close as $5 \lambda_{0} / D\left(2 \lambda_{0} / D\right)$ with a it SNR of 3 .

We then recorded spectra under the same experimental conditions. Curves that are normalized to the non-coronagraphic spectrum are drawn in Fig. 11. On the optical axis (black full line), the MFQPM attenuates the source and leaves a residual energy as low as $3.2 \times 10^{-4}$ from 660 to $800 \mathrm{~nm}$ (20\% bandwidth, see Table 4 ). At $5 \lambda_{0} / D$ (red dashed line), the instrument can record a $2 \times 10^{-4}$ companion spectrum over the $30 \%$ band $(600$ $870 \mathrm{~nm}$ ) with a it SNR of 3. Again, that performance is extracted from the raw coronagraphic data and, as the image quality is limited by speckle noise further than a few $\lambda_{0} / D$ (Fig. 8 ), better contrasts can be reached by applying speckle calibrations.

\section{Conclusions}

We presented the current multi-stage four-quadrant phase mask (MFQPM, Baudoz et al. 2008) laboratory performance. 


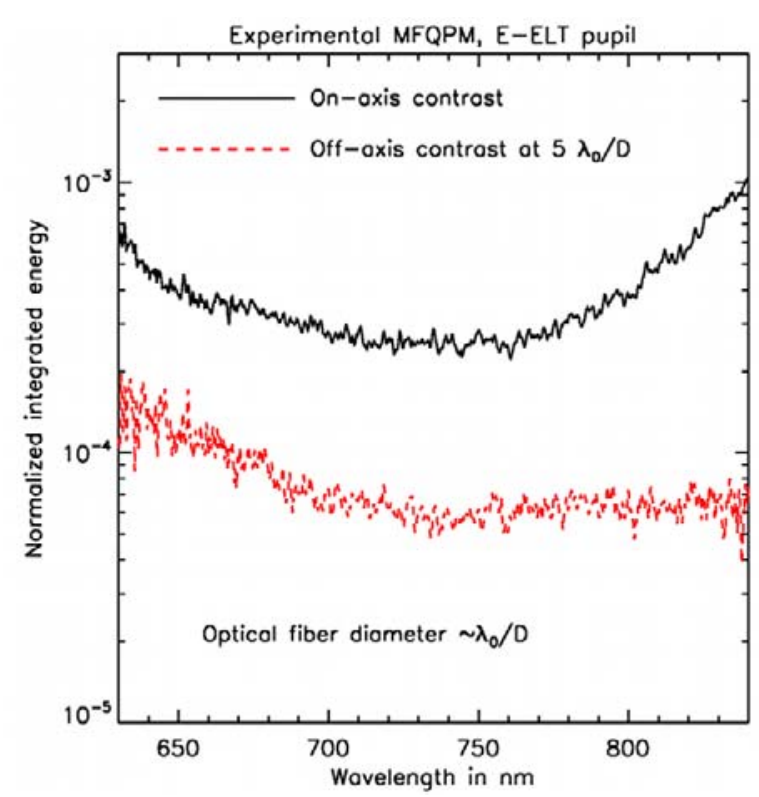

Fig. 11. Experimental residual energy spectral evolution integrated by the spectrometer fiber on the optical axis (black full line) and at $5 \lambda_{0} / D$ (red dashed line) in MFQPM focal plane with E-ELT pupil. The curves are normalized to the non-coronagraphic case.

The instrument uses several monochromatic four-quadrant phase masks (FQPM Rouan et al. 2000) in series. It is easy to build because the monochromatic FQPM fabrication is very well under control since the developments made for JWST and VLT instruments.

In the laboratory, we demonstrated that the MFQPM strongly attenuates the central source over a very wide spectral range $(>20 \%)$ with a high throughput $(86 \%)$ even as close as $2 \lambda_{0} / D$ from the star. Using a $f / 40$ off-axis telescope (full pupil), the actual prototype can record a $2 \times 10^{-6}$ planet spectrum at $5 \lambda_{0} / D$ from its star over a $20 \%$ bandwidth $(660-800 \mathrm{~nm})$ with a $S N R$ of 3.

We also proved that the MFQPM is not very sensitive to ground telescope central obstructions and spiders unlike a lot of coronagraphs. Using an E-ELT pupil and a $f / 40$ optical aperture, the actual prototype can measure a $2 \times 10^{-4}$ planet spectrum at $5 \lambda_{0} / D$ over a $20 \%$ bandwidth with a it SNR of 3.

Finally, it is very important to recall that all these results of the raw MFQPM performance were made under the current laboratory conditions (no cleanroom). As explained in Sects. 3.2 and 3.3, actual limitations are dusts and FQPM transition thickness and phase aberrations at a lower level. To reach higher contrasts (up to $10^{10}$ at a few $\lambda_{0} / D$ ), a new prototype is under fabrication and we will use it in a cleanroom. We will also associate the MFQPM with a self-coherent camera (Baudoz et al. 2006a; Galicher et al. 2010) to both actively (deformable mirror) and a posteriori reduce the speckle noise.

\section{Appendix A: Light distribution in a FQPM Lyot-stop}

The focal plane mask coronagraph effect in the output pupil plane at the wavelength $\lambda$ can be described by

$\psi_{\lambda}^{\prime}(\xi, v)=F\left[F^{-1}\left[\psi_{\lambda}\right] M_{\lambda}(x, y)\right] L(\xi, v)$,

with $F$ and $F^{-1}$ the Fourier transform and its inverse, $\psi_{\lambda}$ and $\psi_{\lambda}^{\prime}$ the pupil plane complex amplitudes before and after the focal mask $M_{\lambda}, L$ the Lyot-stop function, $(\xi, v)$ the pupil coordinates, and $(x, y)$ the focal plane coordinates. The perfect FQPM mask function optimized for the wavelength $\lambda_{1}$ but working at an other wavelength $\lambda$ can be described by

$M_{\lambda}(x, y)=\exp [\mathrm{i} \phi(\lambda)(1-\operatorname{sgn}(x) \operatorname{sgn}(y)) / 2]$,

where $\operatorname{sgn}(x)$ is the sign of $x$ and $\phi(\lambda)=\pi \lambda_{1} / \lambda$ is the phase shift induced between the quadrants. Developing Eq. (A.2), we find

$$
\begin{aligned}
M_{\lambda}(x, y)= & \exp [\mathrm{i} \phi(\lambda) / 2](\cos (\phi(\lambda) / 2) \\
& -\mathrm{i} \operatorname{sgn}(x) \operatorname{sgn}(y) \sin (\phi(\lambda) / 2)),
\end{aligned}
$$

we deduce the field in the output pupil for the wavelength $\lambda$ from Eqs. (A.1) and (A.3)

$$
\begin{gathered}
\psi_{\lambda}^{\prime}(\xi, v)=\exp [\mathrm{i} \phi(\lambda) / 2]\left[\cos (\phi(\lambda) / 2) \psi_{\lambda}(\xi, \nu) L(\xi, v)\right. \\
\left.-\mathrm{i} \sin (\phi(\lambda) / 2) F\left[F^{-1}\left[\psi_{\lambda}\right] \operatorname{sgn}(x) \operatorname{sgn}(y)\right] L(\xi, v)\right] .
\end{gathered}
$$

We recognize two terms multiplied by the phasor $\exp [\mathrm{i} \phi(\lambda) / 2]$. The first term describes a pupil equal to the entrance pupil up to a factor of proportionality and a possible undersizing induced by the Lyot-stop. It corresponds to the light that is not affected by the focal mask. It goes to zero as the wavelength tends to $\lambda_{1}$ ( $\phi$ tends to $\pi$ ). The second term represents the pupil affected by a perfect FQPM weighted by a constant. Assuming perfect optics, an infinite field and an unobstructed pupil, the term is nulled (Abe et al. 2003). In that case, we find

$\psi_{\lambda}^{\prime}(\xi, v)=\exp [\mathrm{i} \phi(\lambda) / 2] \cos (\phi(\lambda) / 2) \psi_{\lambda}(\xi, v) L(\xi, v)$.

The pupil distribution at $\lambda$ after a FQPM optimized at $\lambda_{1}$ is the same as in the entrance pupil but weighted by $\cos \left[\pi \lambda_{1} /(2 \lambda)\right]$. A second FQPM coronagraph can then be applied to reduce this residual light. Considering $n$ coronagraphs in series optimized for $\lambda_{1 \leq i \leq n}$ and no Lyot-stop undersizing, the ratio $\tau$ of the integrated energy with and without MFQPM over the bandwidth $\left[\lambda_{\min }, \lambda_{\max }\right]$ is

$\tau=\int_{\lambda_{\min }}^{\lambda_{\max }} \prod_{i=1}^{i=n} \cos ^{2}\left(\frac{\pi}{2} \frac{\lambda_{i}}{\lambda}\right) \mathrm{d} \lambda$.

\section{References}

Abe, L. 2003, A\&A, 400, 385

Baudoz, P., Boccaletti, A., Baudrand, J., \& Rouan, D. 2006a, IAU Colloq., 200, 553

Baudoz, P., Boccaletti, A., Riaud, P., et al. 2006b, PASP, 118, 765

Baudoz, P., Galicher, R., Baudrand, J., \& Boccaletti, A. 2008, SPIE, 7015

Beuzit, J.-L., Feldt, M., Dohlen, K., et al. 2008, SPIE, 7014

Bloemhof, E. E. 2005, Opt. Exp., 13, 10055

Boccaletti, A., Riaud, P., Baudoz, P., et al. 2004, PASP, 116, 1061

Boccaletti, A., Baudoz, P., Baudrand, J., Reess, J. M., \& Rouan, D. 2005, Adv. Space Res., 36, 1099

Carlotti, A., Ricort, G., \& Aime, C. 2009, A\&A, 504, 663

Galicher, R., Baudoz, P., Rousset, G., Totems, J., \& Mas, M. 2010, A\&A, 509, A31

Kasper, M. E., Alter, G., Thatte, N., et al. 2008, SPIE, 7015

Macintosh, B., Everson, S. A., Bauman, B., et al. 2006, SPIE, 6272

Macintosh, B., Thomas, S. J., Soummer, R., et al. 2008, SPIE, 7015

Mawet, D., Riaud, Z., Absil, O., Surdej, J., et al. 2005, ApJ, 633, 1191

Mawet, D., Riaud, P., Baudrand, J., et al. 2006, A\&A, 448, 801

Mawet, D., Serabyn, E., Kent Wallace, J., \& Pueyo, L. 2011, Opt. Lett., 36, 1506 Riaud, P., Boccaletti, A., Rouan, D., Lemarquis, F., \& Labeyrie, A. 2001, PASP, 113,1145

Riaud, P., Boccaletti, A., Baudrand, J., \& Rouan, D. 2003, PASP, 115, 712

Rouan, D., Riaud, P., Boccaletti, A., Clénet, Y., \& Labeyrie, A. 2000, PASP, 112, 1479

Soummer, R., Dohlen, K., \& Aime, C. 2003, A\&A, 403, 369

Tamura, M., Kandori, R., Kusakabe, N., et al. 2006, SPIE, 6269

Vérinaud, C., Korkiakoski, V., Yaitskova, N., et al. 2008, SPIE, 7014 